Table 1. Partition of oestriol, 6-oxo-oestriol and $6^{6} \alpha^{\prime}$-hydroxyoestriol in the systems ether-water and ethyl acetate-water

\begin{tabular}{lcc} 
& $K=\left(\begin{array}{cc}\text { Ether- } \\
\text { water }\end{array}\right.$ & $\begin{array}{c}\text { Ethyl } \\
\text { conch. in acetate-water }\end{array}$ \\
Oestriol & $7 \cdot 7$ & 24 \\
6-Oxo-oestriol & $0 \cdot 66$ & $5 \cdot 6$ \\
$6^{\prime} \alpha^{\prime}$-Hydroxyoestriol & $0 \cdot 12$ & 0.90 \\
\hline
\end{tabular}

was determined quantitatively in the following way:

The steroid (100-300 $\mu$ g.) spread in a thin film on the inside of a flask was shaken at $25^{\circ}$ with $10 \mathrm{ml}$. of each phase (pre-equilibrated) until all had dissolved. The mixture was then transferred to a separating funnel and, after separation of the phases, $6.0 \mathrm{ml}$. of each was removed and evaporated to dryness in a vacuum desiccator. Each residue was dissolved in $3.0 \mathrm{ml}$. of ethanol and the concentration of steroid determined from the extinction of the solution at $281 \mathrm{~m} \mu$ (oestriol and $6^{6} \alpha^{\prime}$-hydroxyoestriol) or $325 \mathrm{~m} \mu$ (6-oxo-oestriol). The results (Table 1 ) show that the substitution of either an oxo or an ' $\alpha$ '-hydroxyl group at C-6 in oestriol has a surprisingly great effect in increasing the relative solubility in water.

\section{DISCUSSION}

Although neither 6-oxo-oestriol nor 6' $\alpha$ '-hydroxyoestriol has yet been shown to be present in the urine of pregnant women it is by no means improbable that they, or other highly oxygenated oestrogen derivatives with similar distribution characteristics in the systems ether-water and ethyl acetate-water, may be metabolic products of the oestrogenic hormone. In studies on the metabolism of radioactive oestrogens in vivo or in vitro it may therefore be dangerous to draw any conclusions about the nature of the metabolites formed (whether 'free', conjugated, or degraded to acidic substances) on the basis of the extractability of radioactive products from aqueous media by arbitrarily fixed proportions of ether or of other organic solvents (cf. Beer \& Gallagher, 1955; Valcourt, Thayer, Doisy, Elliott \& Doisy, 1955; Jellinck, 1959).

\section{SUMMARY}

1. 6-Oxo-oestriol, $6^{6} \alpha^{\prime}$-hydroxyoestriol and their acetates have been prepared from oestriol by methods based on those of Longwell \& Wintersteiner (1940) and Wintersteiner \& Moore (1959) for the preparation of 6-oxo-oestradiol-17 $\beta$ and $6^{6} \alpha$ '-hydroxyoestradiol-17 $\beta$ from oestradiol-17 $\beta$.

2. Determinations of the distribution of 6-oxooestriol, $6^{\prime} \alpha^{\prime}$-hydroxyoestriol and oestriol in the systems ether-water and ethyl acetate-water showed that the substitution of an oxo or hydroxy group at C.6 in the oestriol molecule has a very great effect in increasing the relative solubility of these derivatives in water.

We gratefully acknowledge the help of Dr J. W. Minnis, who carried out the microanalyses.

\section{REFERENCES}

Bauld, W. S. (1954). Biochem. J. 56, 426.

Beer, C. T. \& Gallagher, T. F. (1955). J. biol. Chem. 214, 335, 351.

Breuer, H., Nocke, L. \& Knuppen, R. (1958). Naturwissenschaften, 45, 397.

Breuer, H., Nocke, L. \& Knuppen, R. (1959). Hoppe-Seyl.Z. 315, 72.

Brown, J. B. (1952). J. Endocrin. 8, 196.

Jellinck, P. H. (1959). Biochem. J. 71, 665.

Loke, K. H., Marrian, G. F. \& Watson, E. J. D. (1959). Biochem. J. 71, 43.

Loke, K. H., Watson, E. J. D. \& Marrian, G. F. (1957). Biochim. biophys. Acta, 26, 230.

Longwell, B. \& Wintersteiner, O. (1940). J. biol. Chem. 133, 219.

Mueller, G. C. \& Rumney, G. (1957). J. Amer. chem. Soc. 79, 1004.

Valcourt, A. J., Thayer, S. A., Doisy, E. A. jun., Elliott, W. H. \& Doisy, E. A. (1955). Endocrinology, 57, 692.

Wintersteiner, O. \& Moore, M. (1959). J. Amer. chem. Soc. $81,442$.

\title{
The Sedimentation of Myosin
}

\author{
By P. JOHNSON AND A. J. ROWE \\ Department of Colloid Science, University of Cambridge
}

(Received 5 June 1959)

A wide range of reported values for the sedimentation coefficient of myosin at infinite dilution, $S_{20}^{0}$ (termed henceforth the extrapolated sedimentation coefficient), exists in the literature. Values of $\mathbf{7 \cdot 3}$ (Snellman \& Tenow, 1948), 7.1 (Weber, 1950), 7.1 (Johnson \& Landolt, 1951), and 6.7s (Mommaerts \& Parrish, 1951) were earlier reported.
More recently values of $6 \cdot 38$ and $6 \cdot 43$ (Holtzer \& Lowey, 1956), 6.15 (Laki \& Carroll, 1955) and 6.20s (Miller, Golder, Eitelman \& Miller, 1952) have been obtained, the last two values referring to low temperatures. Moreover, the work of Laki \& Carroll (1955) and Parrish \& Mommaerts (1954) has suggested that the observed value may be a 
function of the conditions, particularly the temperature and centrifugal field under which it is measured.

The work here reported was designed to investigate in detail the effects of varying temperature, centrifugal field and other experimental conditions on the extrapolated sedimentation constant. In addition to the experiments reported in which the rotor temperature and centrifugal field have been systematically varied, the effect of the buffer solution used, and of method of preparation, including the number of reprecipitations given to the sample, has also been investigated. It is shown that with certain important but understandable exceptions the extrapolated sedimentation constant for myosin is reproducible and independent of the conditions of measurement. A preliminary explanation of the anomalies previously observed and those now reported is given in terms of the molecular transformation of the myosin sample during the course of examination.

\section{MATERIALS AND METHODS}

\section{Materials}

Water. Water used was thrice-distilled in an attempt to remove traces of metal ions and possible surface-active material. A preliminary distillation was followed by two further distillations from Pyrex glass, the first in the presence of permanganate.

Reagents. All reagents used were of A.R. quality.

Buffer solution. The stock buffer solution used, with a $\mathrm{pH}$ of $6 \cdot 7$ and ionic strength $I$ of $0 \cdot 60$, had the following composition (g./l.): $\mathrm{KCl}, 41.0$; $\mathrm{Na}_{2} \mathrm{HPO}_{4}, 12 \mathrm{H}_{2} \mathrm{O}, 4.0$; $\mathrm{KH}_{2} \mathrm{PO}_{4}, 2 \cdot 2$. All experiments were performed in this solution except where otherwise stated.

Myosin. Myosin was prepared from rabbit skeletal muscle by the method of Szent-Gyorgyi (1945), with several minor modifications. After killing and exsanguination, the back muscles were quickly excised and chilled in ice before mincing. All subsequent steps were performed near $0^{\circ}$. The muscle mince was extracted for $10 \mathrm{~min}$. in Guba-Straub solution $(0 \cdot 3 \mathrm{~m}-\mathrm{KCl}, 0 \cdot 15 \mathrm{~m}$-phosphate, $\mathrm{pH}$ $6 \cdot 5)$, with gentle stirring. After removal of residual mince either by centrifuging or straining through muslin, the extract was filtered under slight pressure through a $\frac{3}{4}$ in. pad of paper pulp on a Büchner funnel. Especial care was taken to avoid frothing at this stage. The filtrate was then diluted to $I 0 \cdot 04$, left standing and the supernatant was decanted; the precipitated myosin was brought into solution at $I 0.5$ with solid or $2.4 \mathrm{M}-\mathrm{KCl}$, and allowed to stand for $1 \mathrm{hr}$. to ensure complete solution. After dilution to I 0.28 the small amount of actomyosin present was removed by centrifuging. After two or three reprecipitations at $I 0.04$, the myosin was dialysed against stock buffer solution and stored at $2-4^{\circ}$ in sealed sterile flasks without preservative. Solutions thus obtained were clear, free from 'threads' of denatured protein, and showed a slight opalescence in bulk. No lipid material was noticed in any of the preparations, which is attributed to the pulp filtration stage (cf. Rupp \& Mommaerts, 1957).

\section{Methods}

Sedimentation studies. Ultracentrifuge runs were performed in a Spinco Model $\mathrm{E}$ analytical ultracentrifuge equipped with diagonal-schlieren optics and facilities for continuous temperature measurement and control. Where necessary the rotor was pre-cooled or pre-heated to the desired operating temperature, and the automatic control used to maintain the temperature. Not only was the absolute calibration of the thermistor checked repeatedly against a standard (N.P.L.) thermometer, but the indicated temperature of the rotor in the rotor chamber at the end of an experiment was checked on the external calibrating stand as soon as the rotor was removed from the chamber. This procedure was adopted because it had been found in earlier experiments that appreciable resistance could be built up at or near the mercury-pool contact, giving a temperature error of up to $2^{\circ}$. By frequent renewal of the mercury pool, such errors were avoided.

The absolute accuracy of the final temperature measurements was normally $\pm 0.05^{\circ}$. In all runs the speed of the instrument was obtained by timing the revolution counter over a long interval. Except when otherwise stated, runs were performed at about $59780 \mathrm{rev} . / \mathrm{min}$. and the centrifugal fields reported refer to the inner index position of the cell, assumed to be $5.725 \mathrm{~cm}$. from the axis of rotation at $59780 \mathrm{rev} . / \mathrm{min}$.

A cell with a dural centrepiece was employed for most experiments. Duplicate experiments with a plastic (Kel-F) centrepiece gave identical results, both as regards sedimentation coefficients and measurements of refraction difference. After measuring (on a Pye two-dimensional travelling microscope) the displacement of the maximum boundary ordinate on successive exposures taken at known time intervals, the sedimentation coefficients were evaluated from a plot of $\log x$ against time, where $x$ is the distance from the axis of rotation. The mean magnification factor was calculated for each run by measuring the distance between the reference lines on the photographs. The coefficients obtained were corrected to the viscosity and density of water at $20^{\circ}$ in the usual way (Svedberg \& Pedersen, 1940). A constant value (0.728) for the partial specific volume, $v$ (Parrish \& Mommaerts, 1954), has been assumed over the range $2-26^{\circ}$. After most of this work was complete, $\bar{v}$ was independently determined by pyknometer $\left(10 \mathrm{ml}\right.$.) at $20^{\circ}$ and found to be $0.725 \pm 0.005$. The relative viscosity, obtained by capillary viscometer to \pm 0.001 , and density of the stock buffer solution were determined at $20^{\circ}, 10^{\circ}$ and $5^{\circ}$. Viscosity was calculated for other temperatures by assuming the relative viscosity, $\eta_{\text {rel. }}$, to be independent of temperature over the interval of interpolation. Since $\eta_{\text {rel. }}$ has the values $0.991,0.988$ and 0.977 at $20^{\circ}, 10^{\circ}$ and $5^{\circ}$ respectively, no significant error is involved in this procedure.

Concentration measurements. Nitrogen estimation by the micro-Kjeldahl method (Pregl \& Roth, 1945) has been used as a primary standard, by taking a value of $16.2 \%$ for the nitrogen constant of myosin (Mommaerts \& Parrish, 1951). For routine determinations, a modified biuret estimation has been employed (Rowe, unpublished work). This has been checked against the primary standard for each preparation. Over the range of protein concentration used in this work, it was confirmed that repeated transfers of a protein solution between glass vessels did not cause a 
significant decrease in protein concentration (cf. Rupp \& Mommaerts, 1957).

Area measurements. The areas of schlieren peaks in the sedimentation diagrams were measured by enlarging and tracing the diagrams, and then estimating the enlarged areas either by counting squares or by cutting out the tracings and weighing relative to a standard area; for the latter method, good-quality airmail paper was found to be sufficiently uniform.

Diffusion coefficients. Apparent diffusion coefficients $(B)$, better designated as 'spreading constants' (Parrish \& Mommaerts, 1954), have been calculated by the 'heightarea' method, correcting both total area and maximum height for the fall of concentration during centrifuging.

\section{RESULTS}

Sedimentation under varying conditions at $17 \pm 2^{\circ}$

All freshly prepared samples gave rise to a single well-defined sedimenting boundary in the ultra-

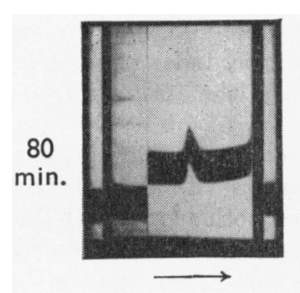

(i)

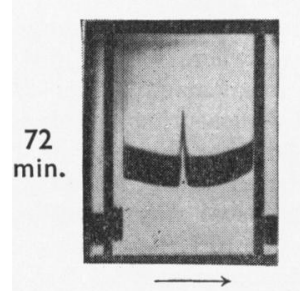

(iii)

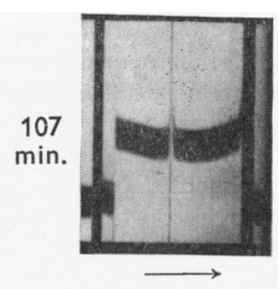

(ii)

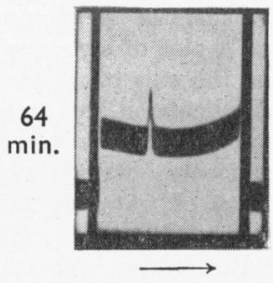

(iv)

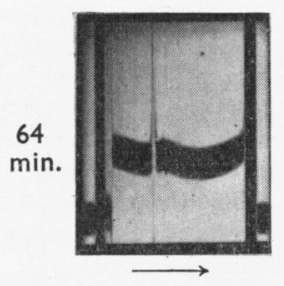

(v)

Fig. 1. Sedimentation diagrams for various myosin preparations [in stock buffer solution except (i)]: (i) Myosin I in $0.5 \mathrm{M}-\mathrm{KCl}$ at $0.156 \mathrm{~g} . / 100 \mathrm{ml}$. and $44770 \mathrm{rev} . / \mathrm{min}$.; (ii) myosin III, 0.31 g./100 ml.; (iii) myosin IV, 0.146 g./ $100 \mathrm{ml}$; (iv) myosin V, 0.17 g./100 ml.; (v) myosin III, as (ii) but stored for 34 days at $24^{\circ}$. Approximate times of sedimentation are given alongside the sedimentation diagrams. centrifuge (Fig. 1, i-iv). More rapidly sedimenting components appeared after storage (Fig. 1, v), as first noted by Weber (1950). Almost all sedimentation runs have therefore been performed within ten days of preparing the sample. Fig. 2 contains collected sedimentation coefficients for several myosin preparations over a range of protein concentration and solvent composition. Table 1 shows the high reproducibility of the extrapolated sedimentation coefficients from four preparations. The regression line, calculated statistically from all the data at $17 \pm 2 \cdot 0^{\circ}$, is given by

$S_{20}^{0}$ (in Svedberg units, $\left.\mathrm{s}\right)=$

$$
6 \cdot 43( \pm 0 \cdot 04)-4 \cdot 3( \pm 0 \cdot 3) c
$$

where $c$ is the concentration in $\mathrm{g} . / 100 \mathrm{ml}$. Standard deviations are given in parentheses. The results obtained in a Kel-F centrepiece are not significantly different (cf. Blum \& Morales, 1953).

Effect of nature of solvent. Experiments were performed with three different, commonly-employed solvents: (i) $0.5 \mathrm{M}-\mathrm{KCl}$; (ii) $0.6 \mathrm{M}-\mathrm{KCl}$; (iii) $0.55 \mathrm{M}-\mathrm{KCl}$ buffered with phosphate to a total ionic strength $I$ of $0 \cdot 60$. Fig. 2 illustrates the results obtained, the regression line being obtained

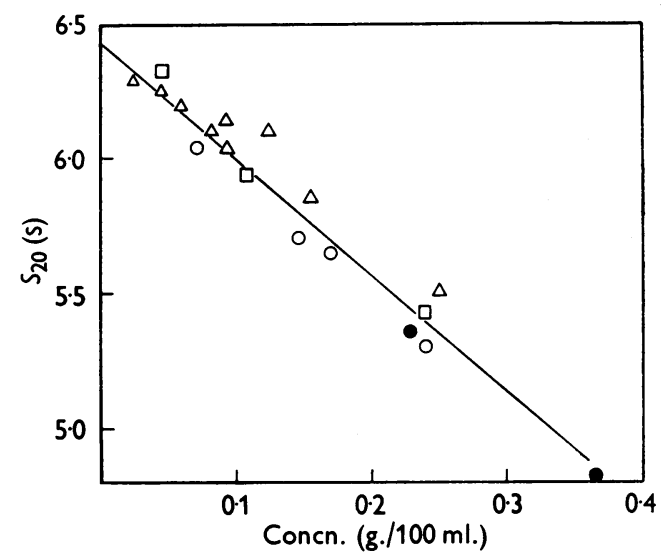

Fig. 2. Sedimentation coefficient (expressed in s) of myosin at $17^{\circ}$ in various solvents. $\triangle, 0.5 \mathrm{M}-\mathrm{KCl} ; \square$, $0.6 \mathrm{M}-\mathrm{KCl}$; $O$, stock buffer solution (KCl-phosphate); , stock buffer solution and Kel-F centrepiece.

Table 1. Extrapolated sedimentation coefficient, $S_{20}^{0}$, for different myosin preparations in stock buffer solution

$\begin{array}{ccc}\text { Number of } \\ \text { Preparation } & \begin{array}{c}\text { Nuprecipi- } \\ \text { tations }\end{array} & S_{: 20}^{0}(\mathrm{~s}) \\ \text { I } & 3 & 6.41^{*} \\ \text { III } & 2 & 6.52 \\ \text { IV } & 2 & 6.36 \\ \text { V } & 0 & 6.41\end{array}$
$\mathrm{KCl}$. 
by the method of least squares, and including all points. It is apparent that any effect of the varying electrolytes must, if present, be small, and certainly less than that required to explain the different values found in the literature.

Effect of reprecipitation of myosin. Snellman \& Gelotte (1951) reported for 'once-crystallized' myosin a value for the extrapolated constant of $7 \cdot 2 \mathrm{~s}$, whereas for heart myosin and twice-precipitated myosin the values of $S_{20}$ at definite concentrations were noticeably lower. A straight-line extrapolation for the latter data (not given by Snellman \& Gelotte) would yield a value of about $6.5 \mathrm{~s}$ for the extrapolated constant. The difference was attributed to a lower actin content in the twice-precipitated sample.

Since it seemed possible that the number of reprecipitations employed could influence the observed sedimentation coefficient, a sample of onceprecipitated myosin was prepared and examined. A plot of observed $S_{20}$ values against concentration yielded a value of $6.41 \mathrm{~s}$ for the extrapolated coefficient, and a slope not significantly different from that given by equation (1). In Table 1 this result can be compared with the values obtained

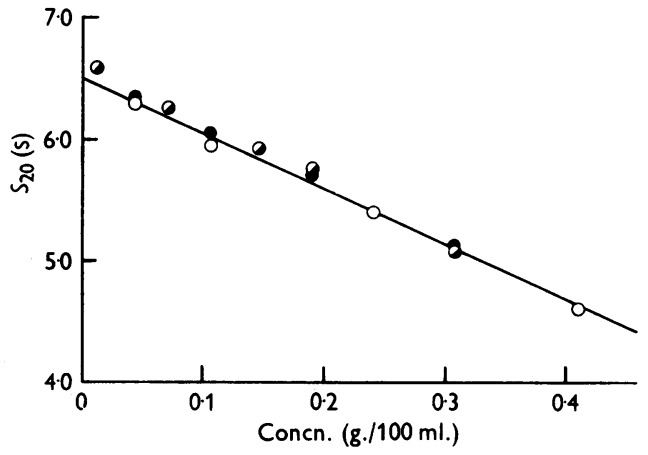

Fig. 3. Sedimentation coefficients (s) of myosin in stock buffer solution at various temperatures: $\bigcirc, 18 \pm 2^{\circ}$; $0,9 \pm 1^{\circ} ; 0,4 \pm 2^{\circ}$.

Table 2. Effect of rise in temperature on the sedimentation coefficient at various concentrations

\begin{tabular}{cccc} 
& & \multicolumn{2}{c}{$\begin{array}{c}\text { Corr. sedimentation } \\
\text { coeff. (s) }\end{array}$} \\
Preparation & $\begin{array}{c}\text { Concn. } \\
\text { (g./100 ml.) }\end{array}$ & $17^{\circ}$ & $25^{\circ}$ \\
III & $0 \cdot 24$ & $5 \cdot 31^{*}$ & $5 \cdot 95$ \\
& $0 \cdot 143$ & $5 \cdot 70^{*}$ & $5 \cdot 87$ \\
IV & $0 \cdot 356$ & $4 \cdot 86$ & $5 \cdot 38$ \\
& $0 \cdot 181$ & $5 \cdot 66$ & $5 \cdot 89$ \\
VIII & $0 \cdot 292$ & $5 \cdot 16$ & $5 \cdot 09$ \\
& $0 \cdot 146$ & $5 \cdot 80$ & $5 \cdot 70$
\end{tabular}

* By interpolation from $S-c$ curve. for other samples, whose preparation involved 1-3 reprecipitations. No significant effect of reprecipitation can be discerned.

\section{Sedimentation at $2-10^{\circ}$}

Fig. 3 illustrates the results obtained for the sedimentation of a myosin preparation at $4^{\circ}$ and $9^{\circ}$, compared with those obtained at room temperature. When the size of the correction factors (up to $80 \%$ for the lowest temperatures) is taken into account, the agreement between corrected $S_{20}$ values for the three temperatures is excellent. It may be concluded that over this range of temperature, and under these conditions, no significant effect of temperature upon the corrected sedimentation coefficient of myosin exists. This view has been confirmed at single concentration values for other preparations. Only with one preparation was an anomalous value, $S_{20}^{0}=7 \cdot 1 \mathrm{~s}$ at $9^{\circ}$, obtained. There is no certain explanation for this single discrepancy, thought it may be significant that this preparation was unusually labile, as judged by the time required for the appearance of fastersedimenting components.

\section{Sedimentation at 24-26 ${ }^{\circ}$}

Anomalously high sedimentation coefficients were obtained over this range for certain preparations (Table 2).

The increases in $S_{20}$ obtained with preparations III and IV, though not large, are significant. Considerable variation from one preparation to another is evident, and the data for any particular preparation were in general less consistent than those obtained at lower temperatures.

\section{Sedimentation at different centrifugal fields}

Experiments were performed at constant protein concentration with speeds varying from 20420 to $59780 \mathrm{rev} . / \mathrm{min}$., giving a ninefold variation in centrifugal field. Parrish \& Mommaerts (1954) originally reported that the effect of centrifugal field on $S_{20}$ was greatest at higher temperatures. Experiments were therefore performed at $25 \pm 1^{\circ}$ in order to accentuate any effect which might be found.

Fig. 4 illustrates typical results obtained. A definite effect of field on observed sedimentation coefficient, especially marked at fields below $100000 \mathrm{~g}\left(\omega^{2}=2 \times 10^{7}\right)$, is evident. Experiments with two other preparations confirmed the very considerable effect at low fields and the almost negligible effect at fields in excess of $100000 \mathrm{~g}$.

\section{Effect of molecular transformation}

It is seen from the above data that the extrapolated sedimentation constant of myosin is a welldefined parameter under a range of conditions at 


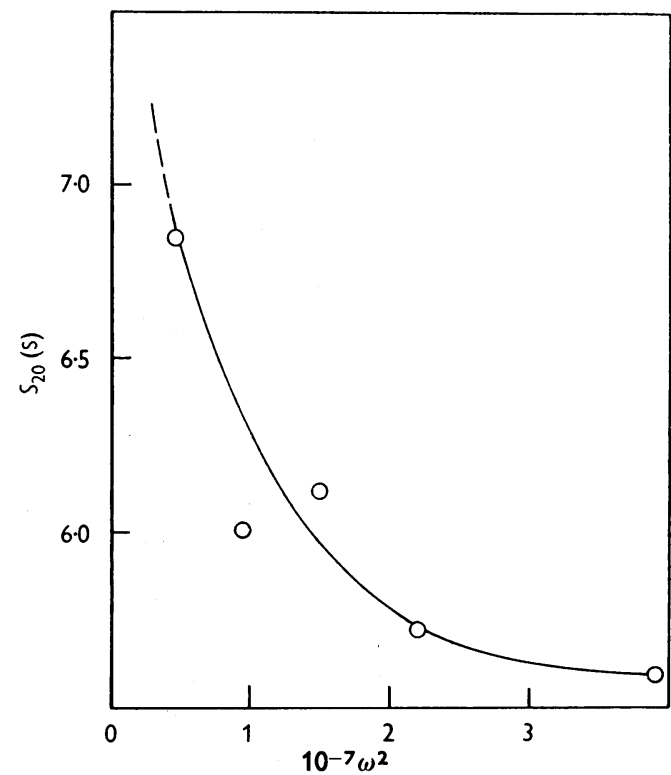

Fig. 4. Sedimentation coefficient (s) of a sample of myosin in stock buffer solution as a function of centrifugal field (concn., 0.146 g./100 ml.; temp., $25 \pm 1^{\circ}$ ).

temperatures below $20^{\circ}$. At higher temperatures increased values are obtained, the increase being small and variable in amount at high centrifugal fields, but pronounced at low centrifugal fields. These conclusions are substantially in accordance with the more recent data published by Mommaerts \& Aldrich (1958). The question arises whether the anomalous behaviour at the higher temperatures can be explained in terms of the known properties of myosin, without postulating a dependence on temperature or centrifugal field of the sedimentation coefficient of the native protein.

Since it is known (Weber, 1950; Spicer \& Weise, 1955; Holtzer, 1956) that myosin can give rise to species sedimenting more rapidly, and that the velocity of this reaction is strongly temperaturedependent, an explanation in terms of this myosin transformation seems possible. There are two extreme sets of conditions under which the formation of components of high sedimentation coefficient could give rise to the observed effects in a solution which initially contains unaltered myosin.

(1) Under conditions of high resolution for the system (that is with maximal centrifugal field and path length in the cell), faster components would become separated from the main sedimenting boundary and travel ahead.

A fall in protein concentration at the main sedimenting boundary would result, causing more rapid sedimentation than would correspond to the initial total myosin concentration. Experimental points would therefore lie above the normal $S_{20}-c$ curve. Some consequences of these conditions applying are: (a) Since the transformation into faster components must be continuous throughout the run, the formation of a discrete faster boundary would not necessarily be expected; rather there would be a slight raising of the base line in front of the main peak in the schlieren diagram, which might or might not be detectable. (b) The corrected areas of myosin peaks as observed in the schlieren diagram should decrease in magnitude during the course of a run at higher temperatures. (c) The observed increase in corrected sedimentation coefficient over low-temperature values should be small, and in no case should values greater than the extrapolated low-temperature constant be obtained. (d) Calculated values for $S_{20}$ should increase continuously during a run. (2) Under conditions of low resolution for the system (with small centrifugal fields and short path length in the cell), different consequences follow: (a) Interdiffusion of the myosin and transformed components would maintain a constant concentration (apart from radial dilution) at the broad sedimenting boundary, and this would be reflected in the constancy of corrected peak areas measured from sedimentation diagrams. (b) The position of the maximum ordinate of the resultant schlieren peak would be a complex function of the relative concentration, diffusion coefficients and concentration dependence of the kinetic properties of the various components present. (c) Although the different components of the boundary would not be resolved under these conditions, and the corrected schlieren peak area would remain constant, the change in composition should give rise to a boundary spreading more rapid than could be accounted for in terms of the diffusion coefficient of native myosin. This might, however, be obscured by the boundary-sharpening process arising from the concentration-dependence of the sedimentation constant. (d) The values for $S_{20}$ should again tend to increase during a run, and could conceivably be higher than the extrapolated low-temperature constant.

However, at both high and low resolutions anomalous effects would be noticeable only at higher temperatures, when an appreciable quantity of myosin is transformed into faster-sedimenting materials during the time taken by an experiment. Then at high centrifugal fields a small increase in $S_{20}$ over low-temperature values would be obtained, while at low fields a much larger effect would be expected. The longer runs required at low fields would make the latter effects even more readily observable.

Experimental data are in detailed agreement with the predictions made above (Figs. 5-8; Table 3). At higher temperatures and high centri- 
fugal fields, the areas of the schlieren peaks decrease during a run, an effect not shown at low temperatures or with low fields (Fig. 5). The schlieren diagrams at $25^{\circ}$ show distinct evidence of faster-sedimenting material ahead of the main peak; over the range $5-17^{\circ}$ no such effect is noticeable (Fig. 6). It was possible to estimate the concentration of unaltered myosin from the mean peak area during a sedimentation experiment and, on plotting $S_{20}$ against corrected concentration, results at $25^{\circ}$ are in good agreement with those at lower temperatures (cf. Fig. 7a,b). Thus the dynamic anomaly reported by Parrish \& Mommaerts (1954) is quantitatively explained.

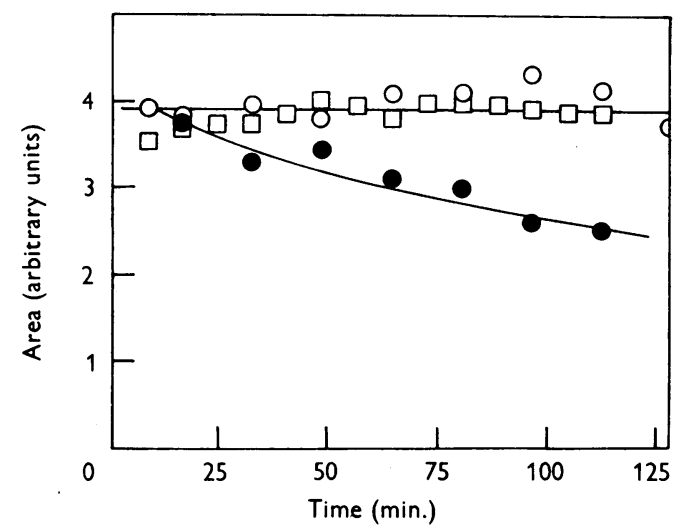

Fig. 5. Schlieren peak area, corrected for radial dilution of myosin $(0 \cdot 146 \mathrm{~g} . / 100 \mathrm{ml}$.), sedimenting in stock buffer solution under various conditions. $\bigcirc$, Temp. $24 \cdot 6^{\circ}$, $\omega^{2}=0.9 \times 10^{7} ; 0$ temp. $20^{\circ}, \omega^{2}=3.9 \times 10^{7} ; \square$, temp. $2 \cdot 2^{\circ}, \omega^{2}=3.9 \times 10^{7}$.

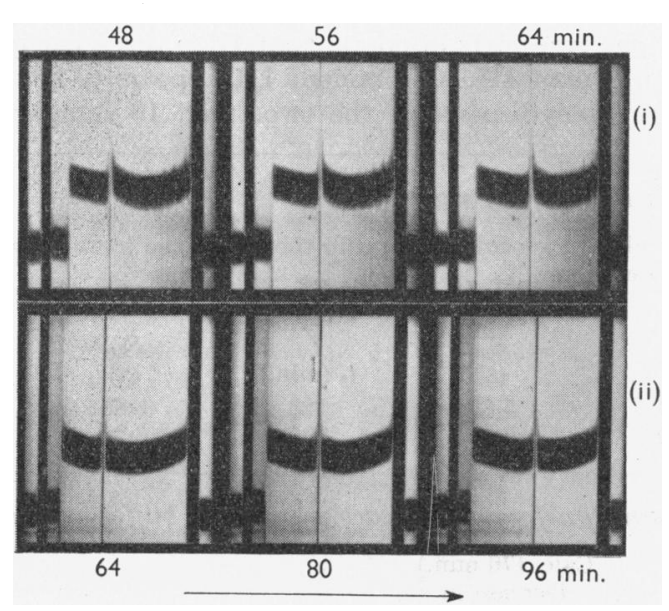

Fig. 6. Sedimentation diagrams for myosin in stock buffer solution at (i) $23.5^{\circ}$, (ii) $17.9^{\circ}$, concn., $0.41 \mathrm{~g} . / 100 \mathrm{ml}$. The approximate time of sedimentation is given for each diagram.
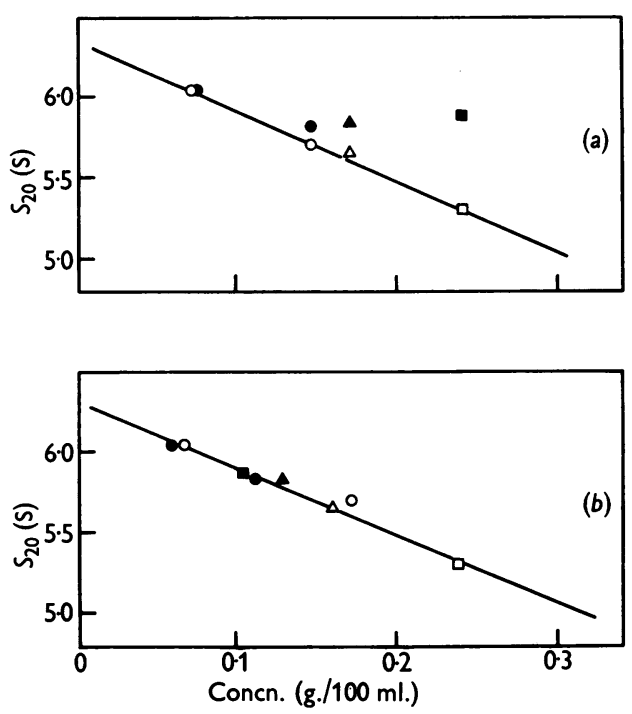

Fig. 7. Sedimentation coefficient (s) of myosin at $17 \pm 2^{\circ}$ (open symbols) and at $25 \pm 1^{\circ}$ (closed symbols) plotted as a function of concentration derived from $(a) \mathrm{Kjeldahl}$ nitrogen estimation, (b) peak-area measurements. Solvents: $O$ and $\odot$, stock buffer; $\triangle$ and $\Delta, 0.6 \mathrm{M}-\mathrm{KCl}$; $\square$ and $\square, 0.5 \mathrm{M}-\mathrm{KCl}$.

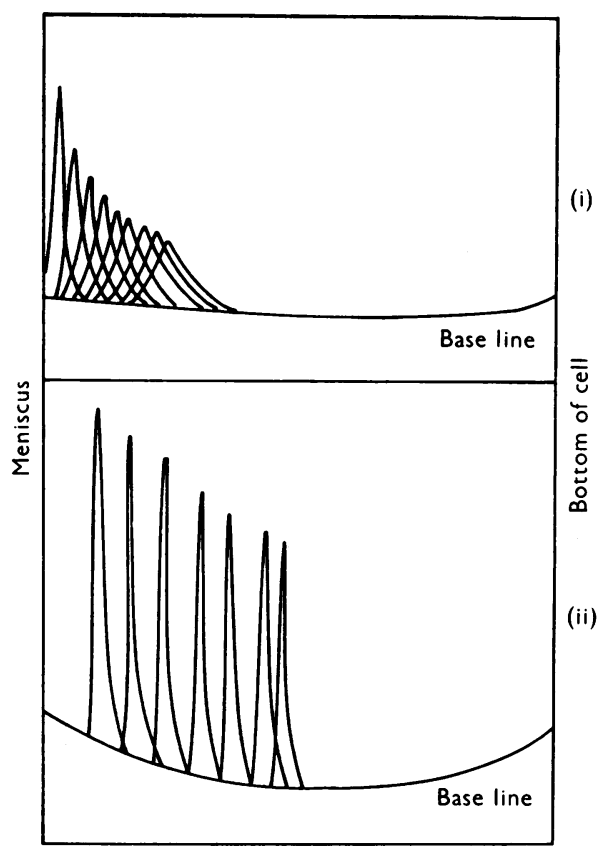

Fig. 8. Boundary spreading during ultracentrifuging of myosin solutions: (i) temp. $24 \cdot 6^{\circ}, 0.146 \mathrm{~g} . / 100 \mathrm{ml}$., $\omega^{2}=0.9 \times 10^{7}$; (ii) temp. $4.5^{\circ}, 0.17 \mathrm{~g} . / 100 \mathrm{ml}$., $\omega^{2}=3.9 \times$ $10^{7}$. Enlarged tracings correspond to $16 \mathrm{~min}$. intervals. 
In Fig. 8 , boundary spreading at $24^{\circ}$ and low speed is compared with that at high speed and low temperature. Although in both cases the total area corrected for radial dilution at the boundary remains constant, the apparent rate of diffusion is found to be considerably greater in the former case $\left(B_{20}=9.02 \times 10^{-8}\right.$ c.g.s. unit) than in the latter $\left(B_{20}=6.5 \times 10^{-8}\right.$ c.g.s. unit), indicating the occurrence of pronounced boundary spreading due to increasing polydispersity at the higher temperature.

Table 3 illustrates the change in sedimentation rate during the course of a run. As would be expected, this is significant only for the low-speed run at high temperature. Because of the inevitable time of standing of the solution during evacuation of the rotor chamber and acceleration of the rotor, together with the need to consider a range of several exposures in order to obtain a reliable value for the sedimentation coefficient, only about a third of the total expected change could be studied by this method. Since at $59780 \mathrm{rev} . / \mathrm{min}$. even the most labile preparations seldom gave an $S_{20}^{0}$ greater than the low-temperature value by more than $0.3 \mathrm{~s}$ (see Table 2), an increase of $0.1 \mathrm{~s}$ (at the most) could be expected during the course of a high-speed run. Much more appreciable increases were, however, observed at low speed.

It remained to confirm that: $(a)$ the rate of formation of transformed products from myosin was such that an appreciable proportion of the protein would be transformed during the time required for a sedimentation run; $(b)$ the difference in sedimentation coefficient between myosin and its transformation products was sufficient to account for the observed behaviour. Such confirmation has been obtained in a detailed study of the heat-transformation reaction of myosin (unpublished work). The reaction has been found to follow first-order kinetics over much of its course, and reaction constants have been evaluated for various conditions. Table 4 gives values for halflife times, $t_{\frac{1}{2}}$, for various preparations at different temperatures (columns 2 and 3 ), and column 4 gives the calculated percentage decrease in myosin concentration occurring in $70 \mathrm{~min}$. at $25^{\circ}$ as a result of the transformation reaction. Because of the finite time needed for resolution of faster components, the actual dilution at the boundary must in all cases be less than the maximal values quoted.

The variation in stability between different preparations is noticeable (compare last columns of Table 4 with Table 2). Sedimentation coefficients have usually been evaluated at a mean time corresponding to about $70 \mathrm{~min}$. after insertion of the sample into the cell. Bearing this in mind, the values for $t_{1}$ in Table 4 confirm that no effect would be expected at room temperature $\left(17^{\circ}\right)$ or below, and that with a particularly stable preparation, such as myosin VIII, little effect would be discernible at high speeds, the lowered concentration at the sedimenting boundary (even at $25^{\circ}$ ) being insufficient to give rise to a measurable rise in $S_{20}$.

It has been suggested (S. Lowey, personal communication) that the presence of phosphate in the buffer causes retardation of the transformation reaction, but in the present work no such effect could be confirmed. Thus the percentage transformation in stock buffer at $25^{\circ}$ in $2 \mathrm{hr}$. of a myosin solution containing $0.24 \mathrm{~g} . / 100 \mathrm{ml}$. was 24 , as against 22 in $0.6 \mathrm{M}-\mathrm{KCl}$.

The heat transformation of myosin regularly gives rise to two distinct faster-sedimenting species, peak II and peak III (see Fig. 9). The relative amounts of components II and III are estimated as about two parts of component II to three parts of component III. Component III appears to be the more polydisperse of the two. Fig. 10 shows the

\section{Table 3. Changes of sedimentation rate during sedimentation}

Concn. of protein, $0.2 \mathrm{~g} .100 \mathrm{ml} . t_{1}, t_{2}$ represent the times after placing ice-cold solution in the cell at the temperatures given in the third column.

$\begin{array}{ccccccc} & \begin{array}{c}\text { Centrifugal } \\ \text { field }\end{array} & & & & & S_{20} \\ \text { Preparation } & (g) & \text { Temp. } & t_{1}(\min .) & (\mathrm{s}) & t_{2}(\min .) & (\mathrm{s}) \\ \text { III } & 228000 & 25 \cdot 2^{\circ} & 33 & 5 \cdot 83 & 123 & 5 \cdot 85 \\ \text { VIII } & 26700 & 24 \cdot 8^{\circ} & 33 & \mathbf{5 \cdot 9 6} & \mathbf{3 0 5} & \mathbf{6 \cdot 2 6}\end{array}$

Table 4. Half-life times $\left(t_{\mathbf{1}}\right)$ and allied data for the myosin transformation reaction in stock buffer solution

\begin{tabular}{lrrcc} 
& \multicolumn{3}{c}{$\begin{array}{c}\text { Calc. (70 min.) } \\
\text { decrease in } \\
\text { concn. at } 25^{\circ}\end{array}$} & $\begin{array}{c}S_{20} \text { at } 25^{\circ} \text { and } \\
\text { Preparation }\end{array}$ \\
\cline { 2 - 3 } & $17^{\circ}$ & $25^{\circ}$ & $(\%)$ & 59 780 rev./min. \\
III & 1156 & 100 & 38 & High \\
IV & 605 & 65 & 53 & High \\
VIII & 1800 & 184 & 23 & Normal
\end{tabular}


sedimentation coefficients of the two components as a function of protein concentration. In each case the concentration taken has been that of the total protein at the sedimenting boundary, estimated from peak-area measurements. Both components sediment more rapidly than myosin, and their presence even at low concentration is adequate to explain the observed increases in $S_{20}$.

\section{DISCUSSION}

The sedimentation coefficient of myosin at infinite dilution is reproducible under a variety of condi-

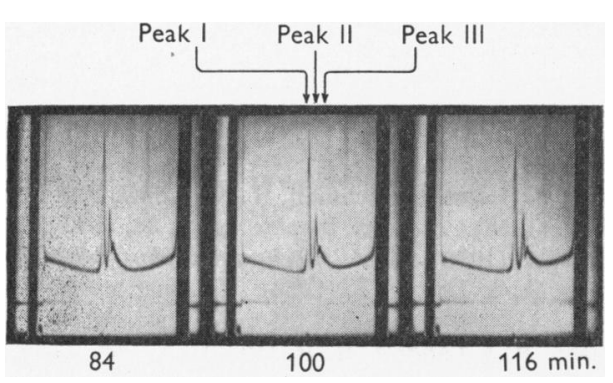

Fig. 9. Sedimentation diagrams for a myosin solution incubated in stock buffer for $183 \mathrm{~min}$. at $25^{\circ}$ before sedimentation at $18-20^{\circ}$ and $59780 \mathrm{rev} . / \mathrm{min}$. Protein concn., $0.40 \mathrm{~g} . / 100 \mathrm{ml}$. The approximate time of sedimentation is given for each diagram.

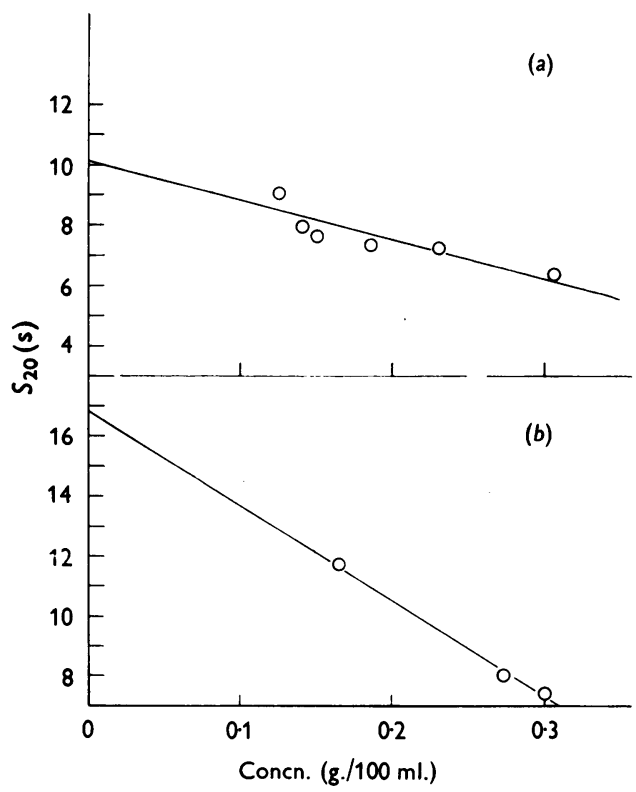

Fig. 10. Sedimentation coefficients (s) of myosin-transformation products [peak II $(a)$ and peak III $(b)$ are as in Fig. 9]. Concentrations are expressed as total protein concentration at the sedimenting boundary. tions, including a wide range of temperature if allowance is made for conversion of myosin into faster-sedimenting species. The value of

$$
S_{20}^{0}=6.43 \pm 0.04 \mathrm{~s}
$$

reported is in good agreement with those reported by Holtzer \& Lowey (1956) of 6.38 and $6.43 \mathrm{~s}$, and that of Hippel, Schachman, Appel \& Morales (1958) of $6 \cdot 25 \pm 0 \cdot 15 \mathrm{~s}$ for the slow component in ' 5 -hr. actomyosin' (i.e. from extraction of muscle mince with Weber-Edsall solution for $5 \mathrm{hr}$.). All these values are much lower than those originally proposed for myosin (Snellman \& Tenow, 1948; Weber, 1950; Johnson \& Landolt, 1950, 1951). It seems probable that the difference is to be accounted for in terms of the transformation reaction, since the earlier values were obtained under conditions of lower resolution (i.e. lower centrifugal field) and higher temperature than those obtaining in recent studies; the sedimentation diagrams of Snellman \& Tenow (1948) suggest the presence of appreciable amounts of transformed material. The formation of an appreciable proportion of transformed material during a run would then be expected to cause a high sedimentation coefficient (cf. Table 2).

The value $S_{20}^{0}=6.66 \mathrm{~s}$ reported by Parrish \& Mommaerts (1954), which requires correction (Waugh \& Yphantis, 1952) for the adiabatic cooling of the rotor (giving $6.83 \mathrm{~s}$ ), is also significantly higher than that reported here, even though it was determined under conditions of high resolution comparable with those employed in the present work. However, the temperature employed was $23 \cdot 6 \pm 1.5^{\circ}$ and, though no quantitative estimate of the amount of transformation can be made, it seems highly probable that this would be appreciable and sufficient to account for the high sedimentation constant reported.

Three reports have suggested that near $0^{\circ}$ a low value for $S_{20}^{0}$ is obtained (Miller et al. 1952; Laki \& Carroll, 1955; Mommaerts \& Aldrich, 1958). These authors obtained values of $6.20 \mathrm{~s}$ at $7^{\circ}, 6 \cdot 15 \mathrm{~s}$ at $4^{\circ}$ and $6 \cdot 10-6 \cdot 20 \mathrm{~s}$ at $6^{\circ}$ respectively, though in no case were detailed results given over a range of concentrations. In the present investigation, no evidence has been found that the corrected sedimentation coefficient is in any way affected by lowered temperature. In reaching this conclusion, particular care has been exercised in confirming the accuracy and reproducibility of the temperature measurements on the ultracentrifuge (p. 433), and it seems quite feasible that faulty operation of the thermistor assembly might be responsible for some of the discrepancies now apparent. The recent suggestion by Mommaerts \& Aldrich (1958) that the correct value for $S_{20}^{0}$ might lie between 5.8 and $6.0 \mathrm{~s}$ is, in the authors' opinion, without foundation. 
Assuming the value 6.43 s for the extrapolated sedimentation coefficient, it is clear that even with Weber's (1950) diffusion coefficient $\left(0.9 \times 10^{-7}\right.$ c.g.s. unit) and his other data, the molecular weight becomes 740000 , considerably smaller than had been earlier visualized. However, it seems likely that the myosin transformation reaction would cause lowered diffusion coefficient as well as raised sedimentation coefficient and the more recent value $\left(1.05 \times 10^{-7}\right)$ of Parrish \& Mommaerts (1954) is probably to be preferred. With $\bar{v}=0.725$ this gives a molecular weight of 540000 . This value is in substantial agreement with light-scattering results, but is considerably larger than recent values obtained by the Archibald technique.

The reported 'dynamic anomalies' of Parrish \& Mommaerts (1954) have been largely confirmed, so far as an apparent effect of higher temperatures and lowered centrifugal fields has been demonstrated. However, such anomalies arise, not from peculiar hydrodynamic properties of the myosin molecule, but from the occurrence of a reaction in which myosin is transformed into faster-sedimenting species in a time which is comparable with that required for a sedimentation-velocity experiment. The agreement between the predicted consequences of such a transformation occurring during sedimentation and the experimental results observed is excellent, within the limits of the present qualitative approach; and there now seems to be little reason for postulating 'dynamic anomalies' in the sedimentation behaviour of native, untransformed myosin solutions.

\section{SUMMARY}

1. The sedimentation of rabbit myosin has been investigated under conditions of varying temperature, centrifugal field and solvent composition.

2. From $4^{\circ}$ to $19^{\circ}$ the corrected sedimentation coefficient is reproducible over a range of solvent composition, and its variation with concentration is given by

$$
S_{20}^{0}(s)=6 \cdot 43( \pm 0 \cdot 04)-4 \cdot 3( \pm 0 \cdot 3) c .
$$

Variation in the number of precipitations of the myosin had no observable effect on the sedimentation behaviour.

3. At $25^{\circ}$ the occurrence of previously reported apparent anomalies has been confirmed. These are small at high centrifugal fields, but relatively large at low fields.

4. Evidence is presented to show that these anomalies may be explained in terms of the transformation of myosin into faster-sedimenting species, without the necessity for assigning unusual hydrodynamic properties to the myosin molecule.

\section{With}

$S_{20}^{0}=6.43 \mathrm{~s}, \quad D_{20}^{0}=1.05 \times 10^{-7}, \quad \bar{v}=0.725$, the calculated molecular weight is 540000 .

One of us (A.J.R.) is indebted to the Medical Research Council for a maintenance grant. We are grateful to $\mathrm{Mr} \mathrm{N}$. Buttress for invaluable assistance with the ultracentrifuge.

\section{REFERENCES}

Blum, J. N. \& Morales, M. F. (1953). Arch. Biochem. Biophys. 43, 208.

Hippel, P. H. von, Schachman, H. K., Appel, P. \& Morales, M. F. (1958). Biochim. biophys. Acta, 28, 504.

Holtzer, A. (1956). Arch. Biochem. Biophys. 64, 507.

Holtzer, A. \& Lowey, S. (1956). J. Amer. chem. Soc. 78, 5955.

Johnson, P. \& Landolt, H. R. (1950). Nature, Lond., 165, 430.

Johnson, P. \& Landolt, H. R. (1951). Disc. Faraday Soc. $11,179$.

Laki, K. \& Carroll, W. R. (1955). Nature, Lond., 175, 389.

Miller, G. L., Golder, R. H., Eitelman, E. S. \& Miller, E. E. (1952). Arch. Biochem. Biophys. 41, 125.

Mommaerts, W. F. H. M. \& Aldrich, B. B. (1958). Biochim. biophys. Acta, 28, 627.

Mommaerts, W. F. H. M. \& Parrish, R. G. (1951). J. biol. Chem. 188, 545.

Parrish, R. G. \& Mommaerts, W. F. H. M. (1954). J. biol. Chem. 209, 901.

Pregl, F. \& Roth, H. (1945). Quantitative Organic Microanalysis, 4th ed. London: J. and A. Churchill Ltd.

Rupp, J. C. \& Mommaerts, W. F. H. M. (1957). J. biol. Chem. 224, 277.

Snellman, O. \& Gelotte, B. (1951). J. exp. Cell Res. 1, 234.

Snellman, O. \& Tenow, M. (1948). Biochim. biophys. Acta, 2, 384.

Spicer, S. S. \& Weise, V. K. (1955). Arch. Biochem. Biophys. 59, 345.

Svedberg, T. \& Pedersen, K. O. (1940). The Ultracentrifuge. Oxford: Clarendon Press.

Szent-Gyorgyi, A. (1945). Acta physiol. scand. 9, Suppl. 25. Waugh, D. F. \& Yphantis, D. A. (1952). Rev. sci. Instrum. 23, 609 .

Weber, H. (1950). Proc. Roy. Soc. B, 137, 50. 